\section{Student mental health; below the radar?}

In 2011, the Royal College of Psychiatrists issued a report on the mental health of students in higher education. The report made recommendations for psychiatrists and the National Health Service, for Higher Education Institutes (HEIs) and a broader category of 'all sectors'.

One of the challenges of working with this potential group of patients is that students may well live within the area of the university for around half the year and elsewhere for the other half. Organisationally, this can create additional problems when compared with those with a fixed address the year round. The report emphasises the importance of access to a dedicated student health service. Across the HEI sector there is a marked variability in terms of the organisation of such services. Indeed, there is a wide range of sometimes disparate bodies involved in the delivery of such services. The report recommends a closer linking of student support services and NHS-based general practitioner and mental health services.

Although the working group that researched and authored the report was disbanded once the report was released. Another working group under the auspices of Universities UK/Guild HE calling for the promotion of 'Mental Well-Being in Higher Education (MWBHE)' continues to campaign for change and in particular this multidisciplinary and multiagency group argues for a greater recognition of mental disorder and suicide awareness among the student body.

With the combination of greater financial pressures on students and a lean graduate employment market there may be an even greater manifestation of underlying vulnerabilities to mental health problems.

There are particular problems for some potentially vulnerable student groups, for example, international students. In the report it is hypothesised that there are four broad ways relating to a majority culture; assimilation, integration, marginalisation and what is referred to as the 'traditional approach' namely the student retains primary identification with their culture and rejects the new culture. It is argued that there is some evidence that such students do best with integration or the traditional approach and worst with marginalisation. In the report, just as is the case in some other institutional settings, for example, prisons, the Christmas period can be an especially difficult time. Universities are oftentimes in large part organised around the standard undergraduate term time. Thus, it becomes important to ensure that there are support services in place particularly outside of that term time. There is a danger of international students being doubly disadvantaged; they experience the isolation of non-term time living alongside a reduction in support services.

We know that suicide rates have gone up over the last 5 years or so and longer among the student body. Despite this, it is not clear that the NHS or HEIs have made nearly enough progress with the majority of the recommendations of the report. As alluded to above, the mental health of students is likely to be more rather than less tested over the coming years with the changes in Higher Education and economic and social challenges that confront us all.

Graham Towl

Correspondence to Professor Graham Towl, PVC and Deputy Warden, Durham University, Palatine Centre, Stockton Road, Durham DH1 3LE, UK; graham.towl@durham.ac.uk

Competing interests None. 